BUFFON NEEDLE METHOD OF

TRACK COUNTING

Raymond Gold, James H. Roberts and Frank H. Ruddy

September 4, 1981

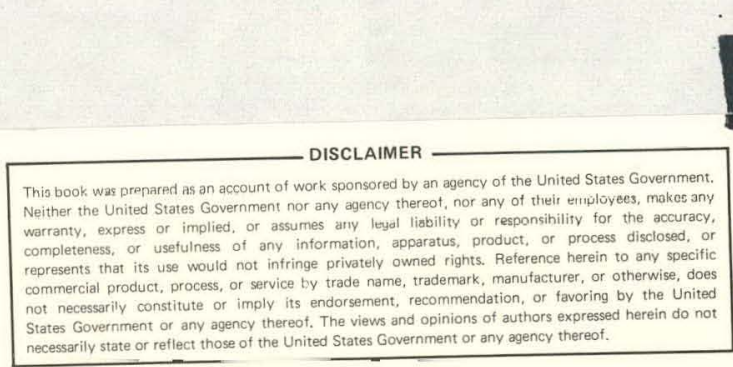

11 th International Symposium

on Solid State Nuclear Track Detectors

September 7-12, 1981

Bristol, ENGI AND

HANFORD ENGINEERING DEVELOPMENT LABORATORY

Operated by Westinghouse Hanford Company, a subsidiary of Westinghouse Electric Corporation, under the Department of

Energy Contract No. DE-AC14-76FF02170 


\section{DISCLAIMER}

This report was prepared as an account of work sponsored by an agency of the United States Government. Neither the United States Government nor any agency Thereof, nor any of their employees, makes any warranty, express or implied, or assumes any legal liability or responsibility for the accuracy, completeness, or usefulness of any information, apparatus, product, or process disclosed, or represents that its use would not infringe privately owned rights. Reference herein to any specific commercial product, process, or service by trade name, trademark, manufacturer, or otherwise does not necessarily constitute or imply its endorsement, recommendation, or favoring by the United States Government or any agency thereof. The views and opinions of authors expressed herein do not necessarily state or reflect those of the United States Government or any agency thereof. 


\section{DISCLAIMER}

Portions of this document may be illegible in electronic image products. Images are produced from the best available original document. 


\title{
BUFFON NEEDLE METHOD OF TRACK COUNTING
}

\author{
Raymond Gold, James H. Roberts, and Frank H. Ruddy \\ Westinghouse Hanford Company \\ Hanford Engineering Development Laboratory \\ Richland, Washington 99352
}

\begin{abstract}
A new technique of quantiative track counting, the Buffon needle method, is advanced. It is based on random sampling of the solid state track recorder (SSTR) surface. This new method extends quantitative track scanning to track densities well up into the track pile-up regime. It is shown that the Buffon needle method possesses a reduced dependence upon both track density non-uniformity and track size distrubition. Sources of experimental error arising in the Buffon needle method are assessed. The validity of the Buffon needle method is demonstrated down to at least the 10\% uncertainty level (lo) by manual sampling of high fission track density mica SSTR observed with scanning electron microscopy.
\end{abstract}

\section{KEYWORDS}

Solid state track recorder (SSTR); track counting; track pile-up; track density; random sampling; Buffon needle.

\section{INTRODUCTION}

Due to the high sensitivity of the SSTR method, track pile-up represents signiticant limitation in quantitative applications. This is especially true for neutron induced reaction rate measurements in high fluence in-situ reactor irradiations (Roberts and Gold, 1977; Gold, Roberts, and Ruddy, 1978; Gold, Ruddy, and Roberts, 1980a; Gold, Ruddy, and Roberts, 1980b).

Pile-up effects are negligible using optical microscopy and manual scanning techniques at track densities lower than $10^{5}$ tracks $/ \mathrm{cm}^{2}$. However, difficulties arise as track density, $\rho$, increases. In fact, for $\rho \geq 2 \cdot 10^{6}$ tracks $/ \mathrm{cm}^{2}$, track pile-up can be extensive enough to preclude quantitative manual scanning using optical microscopy. Even at lower track densities where manual scanning is still possible, experimental error is increased because pile-up decreases the objectivity of manual scanning observations.

Hence, methods or techniques which are capable of extending quantitative track scanning into the high density pile-up region would be quite useful. In particular, methods that can be applied independent of track size are highly desirable. 
Effects of pile-up were recognized in earlier quantitative work with a computercontrolled optical. microscopy system developed for automated SSTR scanning (Gold and Cohn, 1971; Cohn and Gold, 1972; Gold and Cohn, 1972). In these efforts, a useful correspondence was introduced between track scanning and the pulse counting techniques, of nuclear instrumentation. On the basis of this analogy, the simple paralizable counter model was introduced to describe track pile-up and this model provided excellent agreement with experimental results.

The success demonstrated by accurately describing these automated SSTR data confirmed a very simple formula for the probability $P$ of observing tracks without pile-up, namely

$$
p=e^{-\alpha \rho}
$$

where $\alpha$ is the characteristic area for track pile-up. In the theory of stochastic processes (Bharucha-Reid, 1960; Feller, 1966), this exponential holding formula is characteristic of the simplest continuous Markovian stochastic process, namely a Poisson process.

This simple Poisson holding formula forms the basis of a new track scanning method. Equation (1) provides a means to determine the (true) track density in terms of the probability $p$. Hence, rather than attempt to count individual tracks directly, as is customary in manual scanning, one has the alternative of measuring the probability, $p$, and then solving Eq. (1) for $\rho$. One has the simple relation

$$
\rho=-\alpha^{-i} \ln p \text {. }
$$

Since $p$ is the probability of available area (i.e. relative to the total area scanned) for observing tracks without pile-up, it is possible to measure $p$ using random sampling techniques. In this method, one samples the SSTR surface randomiy and tallies whether the area element sampled is occupied by tracks or not. The area element sampled can be chosen to be the mean track area, $\bar{x}$. In the limit of a sufficiently high number of trials, the ratio of unoccupied trails to total trials will approach $p$. This SSTR random sampling technique represents the simplest type of discrete stochastic chain process, namely a Markovian process, since each sample is obviously independent of the outcome of previous sampling results.

This concept was first tested manually by randomly striking into the surface of scanning electron microscope (SEM) microphotographs of fission tracks in mica SSTR with the end of a rod. The area of the end of the rod was chosen to match the mean track area, $X$. To attempt to maintain a random (unbiased) sampling technique, the microphotograph was not vicwed during the striking process. Such a manual random sampling technique possesses an illustrious historical analogue, namely the famous "Buffon needle". problem considered in the 18th century by the Count de Buffon (17071788). Hence Gold, Ruddy, and Roberts (1980c) have called this track counting technique, the Buffon needle method. Correspondingly, the characteristic area parameter, $\alpha$; is called the Buffon area.

An expression for the uncertainty in the track density deduced by the Buffon needle method is easily obtained from Eq. (2). One finds

$$
(\delta \rho / \rho)^{2}=(\delta \alpha / \alpha)^{2}+(1 n p)^{-2}:(\delta p / p)^{2},
$$

where $(\delta \rho / \rho)^{2},(\delta \alpha / \alpha)^{2}$, and $(\delta p / p)^{2}$ are the relative variances of $\rho$, $\alpha$, and $p$, respectively. The binomial probability distribution provides a valid description of random sampling estimates of $p$. Hence, for $n$ random trails, one has for the relative variance of $p$ 


$$
(\delta p / p)^{2}=\frac{p^{-1}-1}{n}
$$

Thus, Eq. (3) becomes

$$
(\delta \rho / \rho)^{2}=(\delta \alpha / \alpha)^{2}+(1 n p)^{-2}\left(p^{-1}-1\right) / n \text {. }
$$

The first term in Eq. (5) represents the error component arising from the uncertainty in the Buffon area $\alpha$. The Buffon area can be determined in a number of different ways. The earlier work of Gold and Cohn (1973) with the computer-controlled optical microscopy system illustrates a method which can, in principle, determine the Buffon area very accurately. Another method, which utilizes the Buffon needle method itself to measure $\alpha$, is illustrated below.

The second term in Eq. (5) provides the error component introduced through random sampling. This expression can be compared with the error that arises in ordinary manual track scanning, where Gold, Roberts, and Armani (1968), have shown that Poisson statistics are applicable. Hence, the relative error in the observation of $n$ tracks by manual scanning is simply $(n)^{-1 / 2}$. Assuming a one-to-one correspondence between the number of random trials and the number of observed tracks, one finds that the factor $\left|(\ln p)^{-1} \cdot\left(p^{-1} \div 1\right)^{1 / 2}\right|$ expresses the deviation of the relative error of random sampling from that of ordinary track counting statistics.

Outside the neighborhood of the two singularities at $p=0$ and $p=1$, the increase in relative error is modest. In fact in the region $0.01 \leq p \leq 0.9$, the increase in relative error ranges from approximately 1.25 up to 3.0. This overall interpretation requires some caution, however, since this comparison tacitly assumes that a single random trial of the Buffon needle method and the manual observation of a single track can be carried out with equal effort. However, this assumption is not generally valid. Indeed for automated systems, random sampling requires considerably less effort.

\section{Effects Due to Non-Uniform Track Density and Track Size Distribution}

In SSTR work, variation of both track density and track size produce limitations in quantitative manual track scanning. Hence it is importent to examine the consequences of non-uniform track density and track size distribution for the Buffon needle method, On the basis of a more general analysis that accounts for both of these effects, it can be shown that

$$
\rho_{0}=\frac{\alpha_{o}}{\sigma_{\alpha}^{2}}\left\{1-\left[1-\frac{\sigma_{\alpha}{ }^{2}}{\alpha_{o}{ }^{2}}\left(\sigma_{\rho}{ }^{2} a_{o}{ }^{2}-2\left[1-v_{\alpha}{ }^{2} u_{\rho}{ }^{2}\right] \cdot \ln \left[\left(1-v_{\alpha}{ }^{2} \sigma_{\rho}{ }^{2}\right)^{1 / 2} \bar{\mu}\right]\right)\right]^{1 / 2}\right\},
$$

where $\rho_{\rho}$ and $\sigma_{\rho}$ are the mean and standard deviation of the track density distribution, respectively ahd $\alpha_{0}$ and $\sigma_{\alpha}$ are the mean and standard deviation of the Buffon area distribution, respectively. Equation $(6)$ can be used to obtain a solution for $\rho_{0}$ in series expansion form. To first order in $\left(\sigma_{\alpha} \sigma_{\rho}\right)^{2}$, one finds

$$
\begin{aligned}
\rho_{0}= & \frac{-\ln \bar{p}}{\alpha_{0}}+\frac{\ln \bar{p}}{\alpha_{0}}\left\{\frac{n_{\alpha}{ }^{2}}{2} \ln \bar{p}-\frac{\eta_{\alpha}^{4}}{2}(\ln \bar{p})^{2}\right\}+\frac{\alpha_{0} \sigma_{\rho}{ }^{2}}{2} \\
& +\frac{\left(\sigma_{\alpha} \sigma_{\rho}\right)^{2}}{2 \alpha_{0}}\left\{1+\ln \bar{p}-n_{\alpha}{ }^{2} \ln \bar{p}-2 n_{\alpha}{ }^{2}(\ln \bar{p})^{2}\right\},
\end{aligned}
$$

where $n_{\alpha}=\sigma_{\alpha} / \alpha_{0}$ and $n_{\rho}=\sigma_{\rho} / \rho_{0}$ are the relative standard deviations of $\alpha$ and $\rho$, respectivefy.

In this series representation, terms have been grouped to correspond to different 
physical effects. Hence the first term represents the simple Buffon needle result

given in Equation. (2) in absence of both effects. The second term in Eq. (7) represents the effect due to the track area distribution alone, whereas the third term represents the effect of the track density distribution alone. The last term in Equation (7) represents the interaction between these two effects, since this term obviously vanishes if either $\sigma_{p} \rightarrow 0$ or $\sigma_{\alpha \rightarrow 0}$. In the special cases whercin either effect is negligible; proper expressions can be obtained by evaluation of Eqs. (6) or (7) in the limit $\sigma_{\alpha} \rightarrow 0$ or $\sigma_{p} \rightarrow 0$.

While the magnitude of the various terms in Equation (7) will obviously depend on the values of $\bar{p}, \alpha_{0}, \sigma_{\alpha}$ and $\sigma_{\rho}$, it is apparent that effects due to non-uniform track density and track size distribution are only of second order. Hence the Buffon needle method possesses the significant advantage of a reduced dependence upon both track density non-uniformity and track size distribution.

\section{SEM APPLICATION OF THE BUFFON NEEDLE METHOD}

Extensive SSTR neutron dosimetry has been conducted in a critical assembly mockup of a Light Water Reactor (LWR) pressure vessel (Ruddy, Gold, and Roberts, 1980). Since results of the highest possible accuracy were desired, all of these SSTR were simultaneously etched using the standardized procedure developed for mica. While most of these SSTR possessed acceptable track density, six of mica SSTR used with asymptotically thick deposits had very high track density. It was decided to use SEM techniques so that these six high track density SSTR could be examined at higher magnification. However even at $2000 x$, pile-up made it difficult to manually scan these SSTR accurately and objectively.

To apply the Buffon needle method to these SSTR, ten microphotographs were taken of each SSTR. With each SSTR, randomly selected surface areas were chosen for these ten SEM microphotographs. Approximately 100 random samples were carried out on each microphotograph, or a total of about 1000 random samples for each SSTR. Measured values of $p$ obtained for these six mica SSTR are summarized in Table 1.

To deduce track densities from these measurements, one must also know a, the Buffon area. In this work, a was measured using the Buffon needle method itself. In this case, Equation (1) is not solved for $\rho$, but rather for $\alpha$. One has

$$
\alpha=-\rho^{-1} \cdot \operatorname{lnp} \text {. }
$$

The uncertainty in this determination of $\alpha$ is given by

$$
(\delta \alpha / \alpha)^{2}=(\delta \rho / \rho)^{2}+(\ln p)^{-2} \cdot\left(\frac{p^{-1}-1}{n}\right) \text {. }
$$

In order to measure $\alpha$ with the Buffon needle method, ten randomly selected SEM microphotographs were taken of a low fission track density mica SSTR. In these measurements, the track density $\rho$ was obtained by directly counting tracks in each of the ten SEM microphotographs. The chosen SSTR possessed a track density low cnough to avoid any pile-up rerngnition problems at the chosen SEM magnification, 2000x. Approximately 100 random samples were taken on each SEM microphotograph. The maan value for the Ruffon Area obtained from these ten SEM microphotographs was $\alpha=(4.58+0.36) \times 10^{-7} \mathrm{~cm}^{2}$.

Track densities for the six high density SSTR were obtained using this Buffon needle measured value of $\alpha$ together with the observed values, $p$, in Equation (2). The resulting track densities, which are given in Table 1 , range from 1.9 to $3.8 \times 10^{6}$ tracks $/ \mathrm{cm}^{2}$. The relative error in these track density measurements is about $10 \%$ and is dominated by the $9 \%$ relative error in $\alpha$. 
TABLE 1 Buffon Needle Track Densities ${ }^{\star}$ from SEM Observations of Mica SSTR

\begin{tabular}{|c|c|c|c|c|c|}
\hline SSTR Dosimeter & $p$ & $\rho$ & م م & $\rho_{v}^{* *}$ & $e_{v} / \rho_{a}$ \\
\hline $23-28-1^{\prime}$ & $0.405 \pm 0.015$ & $2.00 \pm 0.20$ & $2.02 \pm 0.20$ & 2.02 & 1.00 \\
\hline $23-28-2$ & $0.339 \pm 0.013$ & $2.39 \pm 0.23$ & $2.44 \pm 0.23$ & 2.21 & 0.91 \\
\hline $23-28-3^{-}$ & $0.306 \pm 0.014$ & $2.47 \pm 0.24$ & $2.53 \pm 0.24$ & 2.90 & 1.15 \\
\hline $23-28-4$ & $0.288 \pm 0.014$ & $2.76 \pm 0.27$ & $2.84 \pm 0.27$ & 3.28 & 1.15 \\
\hline $23-28-5^{-}$ & $0.422 \pm 0.015$ & $1.91 \pm 0.19$ & $1.93 \pm 0.19$ & 2.00 & 1.04 \\
\hline $24-25-3^{-}$ & $0.178 \pm 0.012$ & $3.82 \pm 0.37$ & $4.01 \pm 0.37$ & 3.66 & 0.91 \\
\hline
\end{tabular}

On the basis of the higher order treatment described by Eqs. (6) and (7), corrected Buffon needle results have been obtained. The corrected Buffon area was $\alpha_{0}=(4.63+0.37) \times 10^{-7} \mathrm{~cm}^{2}$ and corrected $\rho_{0}$ track densities have been included in Table 1. It can be seen that correction for higher order effects ranges from approximately 1 to $5 \%$.

An attempt to confirm these corrected Buffon needle results has been carried out by visually scanning these SEM microphotographs. However, this task is obviously not straightforward. Due to pile-up, the number of tracks observed is often ambiguous. Consequently the objectivity of such observations cannot be guaranteed. Nevertheless, approximately 300 tracks were counted for each of these mica SSTR dosimeters and the corresponding area scanned on the SEM micorphotographs was determined. The visual track density so obtained, $\rho_{v}$, can also. be found in Table 1.

The deviation between visual and corrected Buffon needle track densities is within experimental error for each of these six comparisons. In fact, the observed deviations between visual and corrected Buffon needle track densities averaged over these six SSTR is only about $9 \%$. Hence, this comparison provides confirmation of the validity of the Buffon needle method down to at least the $10 \%$ uncertainty level $(1 \sigma)$.

An alternative comparison can he obtained using the $(\rho v, p)$ data points. A linear least squares fit of the $\rho_{y}$ data as a function of $(-\operatorname{lnp})$ is presented in Fig. 1. This least squares fit verifies the simple Possion holding formula and provides a Buffon area value of $\alpha=(4.55+0.78) \times 10^{-7} \mathrm{~cm}^{2}$. This least squares value of $\alpha$ is in excellent agreement with the Buffon area obtained by random sampling. Consequently, this alternative comparison again confirms the validity of the Buffon needle me thod. 


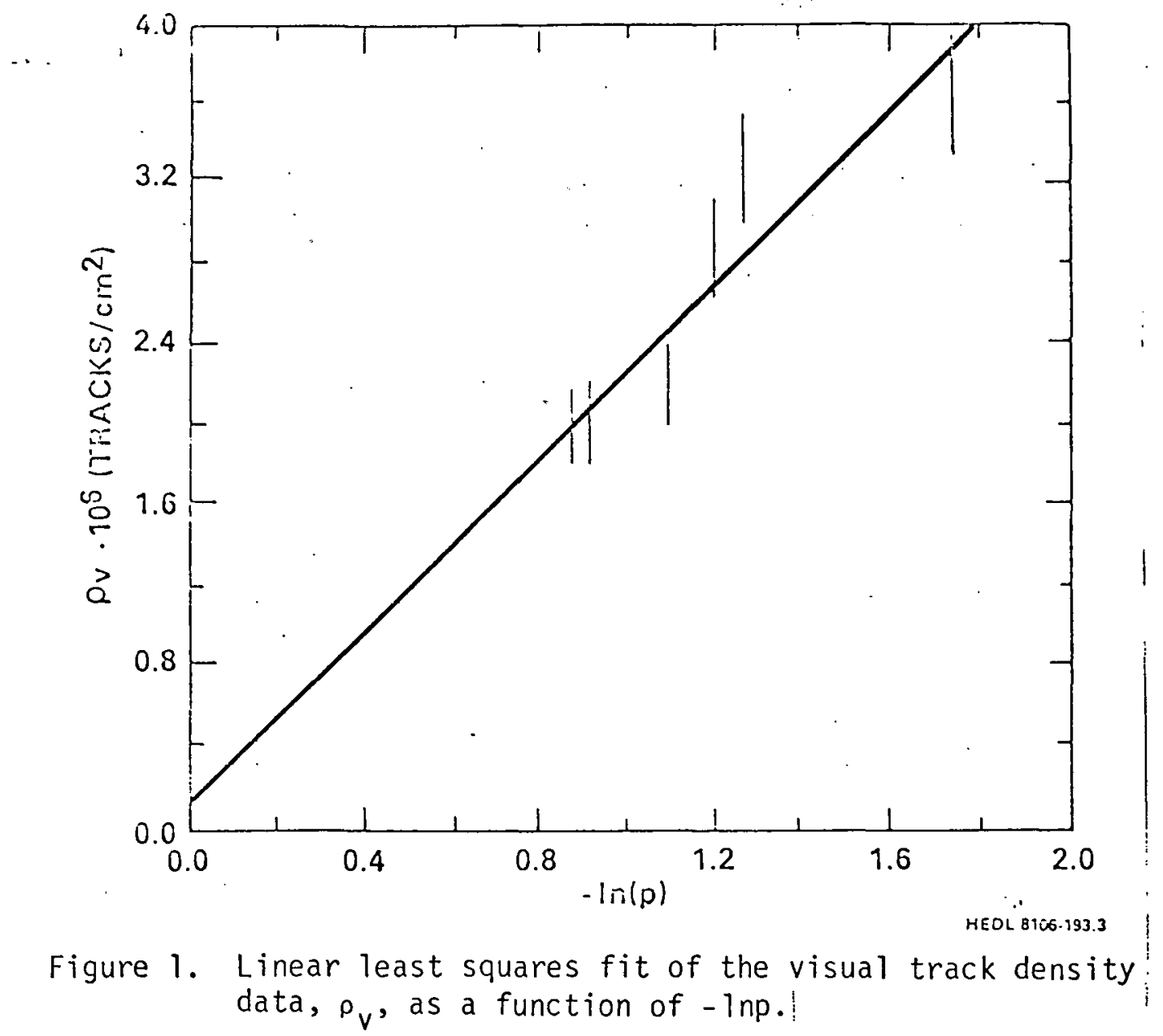

\section{CONCLUSIONS}

The two alternative comparisons presented above confirm the validity of the Buffon needle method. This does not imply that the method is actually applicable without. limitations. Of particular interest is the doma in of track density over which the Buffon needle method is applicable. Efforts to date have confirmed that this method can be used to extend quantitative SSTR scanning by at least an order of magnitude in track density. In spite of these efforts, the domain of validity of the simple Poisson holding formula, which underlies the Buffon needle method, has not been definitively established. Indeed, the concept of a Buffon area a that is independent of track density may possess only a limited range of validity in track density space.

Work is currently underivay to resolve these critical issues. Application of the Buffon needle method with automated SSTR scanning systems is being used in these investigations and this work will be reported in sequel. In this regard, while the Buffon needle method has been demonstrated with manual techniques, the intrinsic advantages of this method for automated track scanning can be expected to produce cven greater benefits for quantitative SSlR work.

Indeed, a major factor limiting the cost-effectiveness of the SSTR method is the necessity of visual or manual counting of tracks, a task which requires care, patience, and dedication. This drawback is clearly manifested in precision measurements, where inherent statistical limitations require the observation of large numbers of tracks for adequate precision and make this task time consuming as we 11 as expensive. As a consequence, automation of this task has been aggressively pursued for some time now. 
As has occurred so often in scientific pursuits, help toward the solution of this automation problem has arisen in the most unexpected way. In this particular case, a completely new approach for automated track scanning has been engendered by the existence of track pile-up at high track density. our efforts to treat this pile-up effect have opened up an entirely new direction for automated track scanning, namely the random sampling techniques of the Buffon needle method. The reduced dependence of the Buffon needle method upon both track density non-uniformity and track size distribution are very important advantages for automated track scanning. However an even more significant advantage exists, namely elimination of the need for pattern recognition of tracks in the automation process. This rather complex procedure can now be replaced, in principle, with the simple random sampling techniques used in the Buffon needle method.

\section{REFERENCES}

Bharucha-Reid, A. T. (1960). Elements of the Theory of Markov Processes and The ir Applications, McGraw Hill, New York.

Cohn, C. E., and R. Gold (1972). A computer-controlled microscope for automatic scanning of solid state nuclear track recorders. Rev. Sci. Instrum., 43, 12-17.

Feller, W. (1966). An Introduction to Probability Theory and Its Applications, Vols. I and II, John Wiley and Sons, New York.

Gold, R., R. J. Armani, and J.H. Roberts (1968). Absolute fission rate measurements with solid atate track recorders. Nucl. Sci. \& Eng., 34, 13-32.

Gold, R., and C. E. Cohn (1971). Analysis of automatic fission track scanning data. Trans. All. Nucl. Soc., 14, 500.

Gold, R., and C. E. Cohn (1972). Analys is of automatic fission track scanning in solid state nuclear track recorders. Rev. Sci. Instrum., 43, 18-28.

Gold, R., J. H. Roberts, and F. H. Ruddy (1978). Solid state track recorder materials for use in light water reactor pressure vessel exposures. In J. A. Sprague and D. Kramer (Eds.) Effects of Radiation on Structural Materials, ASTM STP 683, Amer, Soc. for Testing and Materials, Philadelphia, 402-423.

Gold, R., F. H. Ruddy, and J.H. Roberts (1980a). Application of solid state track recorders in United States nuclear reactor energy programs. In H. Francois and others (Eds.) Solid State Nuclear Track Detectors, Proceedings of the 10th International Conference, Lyon, 2-6 July 1979, Pergamon Press, 0xford, 533-547.

Gold, R., F. H. Ruddy, and J.H. Roberts (1980b). Solid state track recorder applications in U. S. nuclear reactor energy programs. Trans. Am. Nucl. Soc., $34,146-148$.

Gold, R., F. H. Ruddy, and J. H. Roberts (1980c). Buffon needle method of track scanning at high track density. Bu11. Am. Phys. Soc., 25, 484.

Roberts, J. H., and R. Gold (1979). SSTR and emulsion techniques and the ir applications for FBR, LWR, and MFER programs. Dosimetry Methods for Fuels, Cladding, and Structural Materials, Proceedings of the Second ASTM-EURATOM Symposium on Reactor Dosimetry, 3-7 0ctober 1977, NUREG/CP-004, Vol. 2, 739-774.

Ruddy, F. H., R. Gold, and J. H. Roberts (1980). Solid state track recorder measurements in the poolside critical assembly. Dosimetry Methods for Fuels Cladding, and Structural Materials, Proceedings of the Third ASTM-EURATOM Symposium on Reactor Dosimetry, 1-5 0ctober 1979, EUR 6813, Vo1. II, 1069-1075.

This work was supported by the U.S. Department of Energy and the U.S. Nuclear Regulatory Commission. 